\title{
Emergency Centers Set-up in the Existence of Ad Hoc Networks in Disaster Recovery Areas
}

\author{
https://doi.org/10.3991/ijes.v7i1.10319
}

\author{
Saher Manaseer $\left.{ }^{\varpi}\right)$, Afnan Alawneh \\ The University of Jordan, Amman, Jordan \\ saher@ju.edu.jo
}

\begin{abstract}
In disaster recovery situations, rescue teams build portable emergency rooms, fire stations and communication centers in the affected areas. Moreover, Mobile Ad Hoc Networks are the main form of communication that these teams use in such cases. This paper introduces a new setup model for distributing such rescue stations and centers. In this model, the communication needs; play a key role in the decision making to reach the optimal communication performance levels that leads to achieving the goal of using Ad Hoc Networks in these situations.
\end{abstract}

Keywords-Emergency centers, Disasters, MANETS, Mobility model, Hexacells.

\section{Introduction}

\subsection{Applying the styles to an existing paper}

During and after the hazardous situations, the recovery processes are the significant actions to be managed, since people think about the disasters the critical direct moments after the disaster ends or the hazard disappears. Dealing with the consequences of such emergency situations needs good planning taking into consideration the importance of communication channels. The most critical concern about these situations is how to maintain communications from collapsing or having eradicated communications infrastructure [1][2][3].

The decisions in such scenarios are critical, so the information on which such decisions are made shall be accurate and precise to minimize the losses as much as possible. Here comes the importance of planning for a good trusted communication infrastructure-less network (MANETs) [3]. Previously a theoretical framework for MANETs in disasters situations was proposed and an algorithm for nodes propagation was set, in a way that manages the situations in damaged and unreachable areas after the disasters [4].

The proposed mobility model in this paper concentrates on the emergency centers distribution according to best nodes propagation scenario, in a way that helps to avoid losses as well as increasing credibility of MANETs performance in such scenarios. 


\section{Related Works}

Predicting and estimating the severity and happening of the disasters is hard to be controlled. Especially in the cases of earthquakes, hurricanes, volcanoes, attacks etc [2].

In such scenarios, MANETs are the best choice for avoiding broken structures and make a good disaster post management plan [4][5]. The complexity of such protocols is related to the metrics of performance especially the reliability of communication and delays, also the power consumption, these metrics makes building a management system is a complex job [6].

Nodes smart positioning technique was used to introduce the COMVIVOR framework, to be used for survivors in disastrous situations in order to improve the dynamic propagation of the nodes [7].

Time is a very critical element in the cases of disasters, the proposed protocols and algorithms that aim at turning the GPS maps into digital representations suffers the time delays, also the memory space required for processing and saving data [8]. The reliability of collected data is a significant issue in these scenarios. Moreover, the time needed to collect and deliver information is an important issue to take care of [9]. For this the opportunistic network's protocols were developed and proposed [10].

[11] Had introduced an information system for handling server failure in disasters cases by utilizing the cloud computing. In [12] the DistressNet was proposed; which is a wireless sensor network that has the ability to overcome many predefined cases in disasters to collect required information for enhancing the rescue or evacuating processes. In another experiment for generating maps during disasters, a system for generating pedestrians' maps was built; this system does not depend on cloud computing in collecting needed information for maps generating processes [7].

The security issue of many disasters framework models was surveyed in [13], most of proposed work didn't take security into account as a significant issue to solve. The survey focused on privacy, integrity, and authentication of data as major security keys in disasters. The lack of these issue in the proposed protocols in such catastrophic cases can lead to less reliable and fake information. So security service (QoS) shall be counted when proposing protocols for managing emergency and disasters cases.

Exchanging the information between the emergency centers in these scenarios is critical and crucial for it can save many lives. [14] Have studied post disasters management through the location of the disaster, first aid, and location of the emergency center (Hospitals), and the relief staff and ambulance. Using the reference point of group mobility, the model was built. The attraction point was used as a base to build the mobility designs scenarios.

A new scheme for hybrid wireless networks resource management was proposed by [15], the scheme selects some powerful end users to act as temporal hosts, to achieve efficient system resource utilization. The purpose of this scheme is to minimize the blocking probability and increase the parallel connections in disasters management scenarios, through finding a free resource for the requests of connections in this emergent cases. Moreover, the introduced scheme provided fairness in the cases of multiple services scenarios. 
In this paper, the new idea is how to distribute the relief emergency centers, in post disasters management plans, in order to achieve the best distribution that helps in minimizing loses and increase the reliability of rescuing processes, according to the mobility model of Ad hoc networks that was previously proposed in [4].

\subsection{Topology of streets segments}

In order to apply the proposed algorithm in this paper, the street map shall be presented for the disaster area, then the mobility model is applied on the given represented map, after that the proposed algorithm in the paper is applied to find the best points on the map to distribute the relief emergency centers, for minimizing the losses and saving more lives.

The proposed algorithm contains three main steps:

- Step1: find the street segments maps

- Step2: apply the hexagonal mobility model proposed in [4]

- Step3: apply the proposed model in this paper, to distribute the emergency centers.

Streets segments are represented as lines in the sketch maps, the connections are made clear at the crossroads points. When the connectivity to other roads ends, it is called an endpoint.

\subsection{Dipole Relation Algebra (DRA) (Discarding the relative orientation)}

This algorithm considers dipoles, the oriented lines segments for capturing the topology of the streets. The dipole is a pair of ordered points represents the start and the end of a street line respectively, it is represented as (A "The Dipole"= (As, Ae); s=start, $\mathrm{e}=$ end).

The positions of dipoles with respect to each other are $\mathrm{s}$ for start, e for ending, or $\mathrm{x}$ for neither of them. DRA does not take into consideration the orientation of the dipoles at junctions. There are seven available options: sese, eses, xexe, sxsx, xsex, exxs in the case of connectivity, and $\mathrm{xxxx}$ for the segments that are not connected.

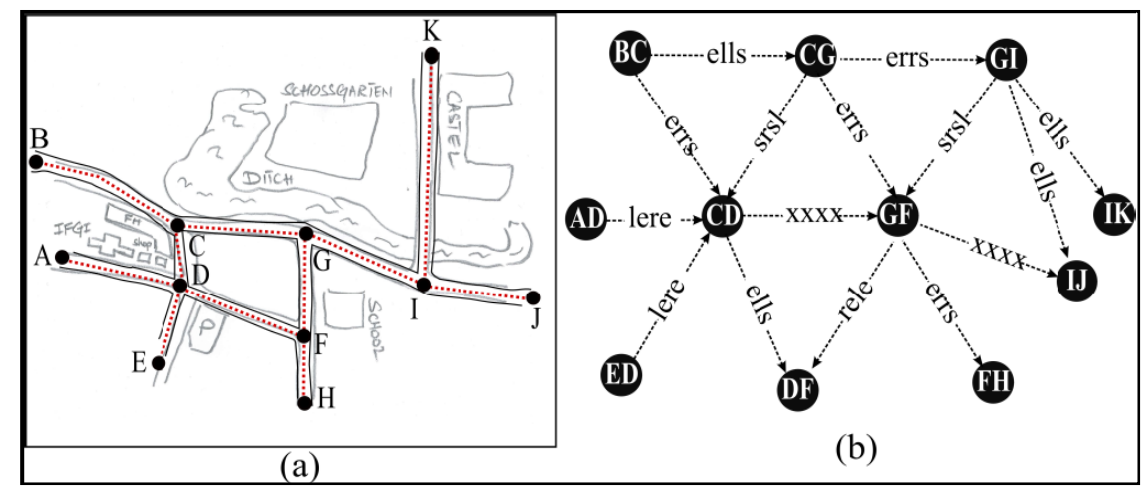

Fig. 1. Dipole Relation Algebra (DRA) 


\subsection{Orientation of street segments}

To solve the problem of distorting angles and straightened curved streets, two qualitative representations were used; The STAR and OPRA calculi.

The following figure shows the orientation implementation using the junctions of the street segments. The points on the junctions' areas are represented with their orientation.

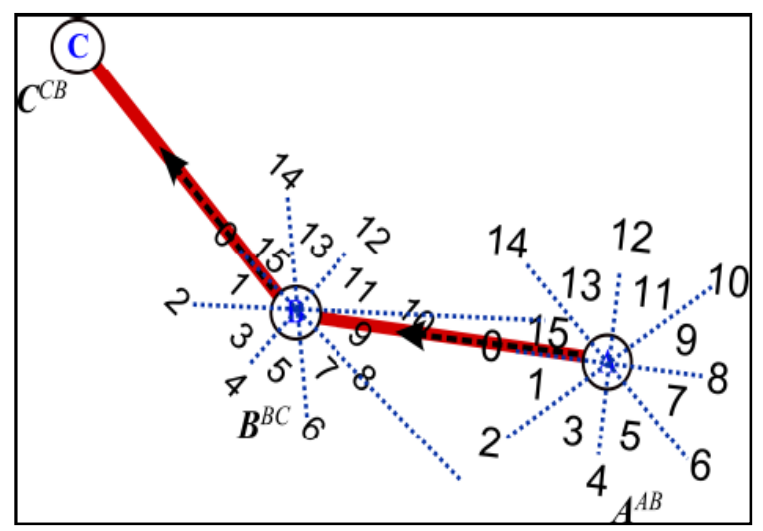

Fig. 2. Orientation of street segments

\subsection{Cluster analysis of angles and orientation sectors}

Clustering works by dividing the angles in the map into a specified number of homogeneous groups. In each cluster of angles, the range is examined and codded as follow:

Angles: $53^{\circ}$ to $155^{\circ}, 112^{\circ}$ to $248^{\circ}$, and $205^{\circ}$ to $305^{\circ}$ [coded as a left, front, and right respectively], the overlapping as the figure shows means that there are some distorted segments are codded into left, while other segments with almost the same range of angles and clusters are coded into front cluster (Half right).

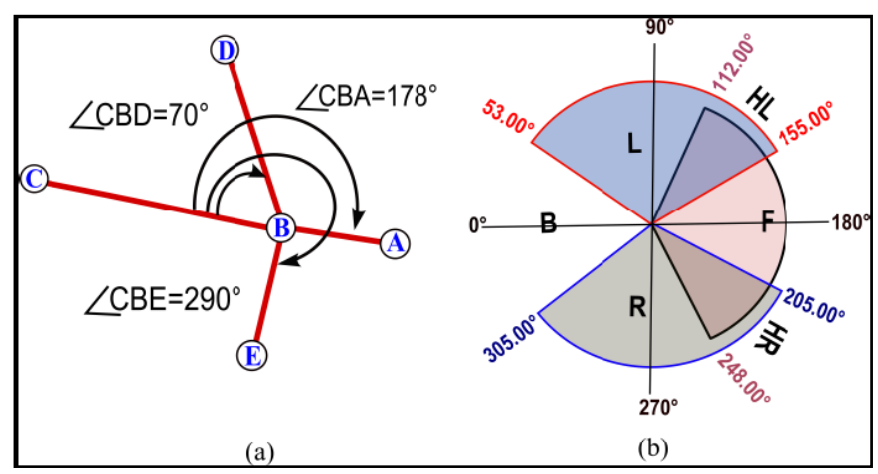

Fig. 3. Cluster Analysis of Angles and Orientation Sectors 


\section{Proposed Model for Emergency Centers Set-up}

The areas affected by any disaster are divided into hexagonal cells as stated in the algorithm proposed in [4], also the border cells' nodes are stated as border nodes and given the highest priority as stated in [16]. As well as the roads segments mentioned in (Topology of Streets Segments).

Proposed Algorithm of [4] for dividing the disaster area into hexa-cells:

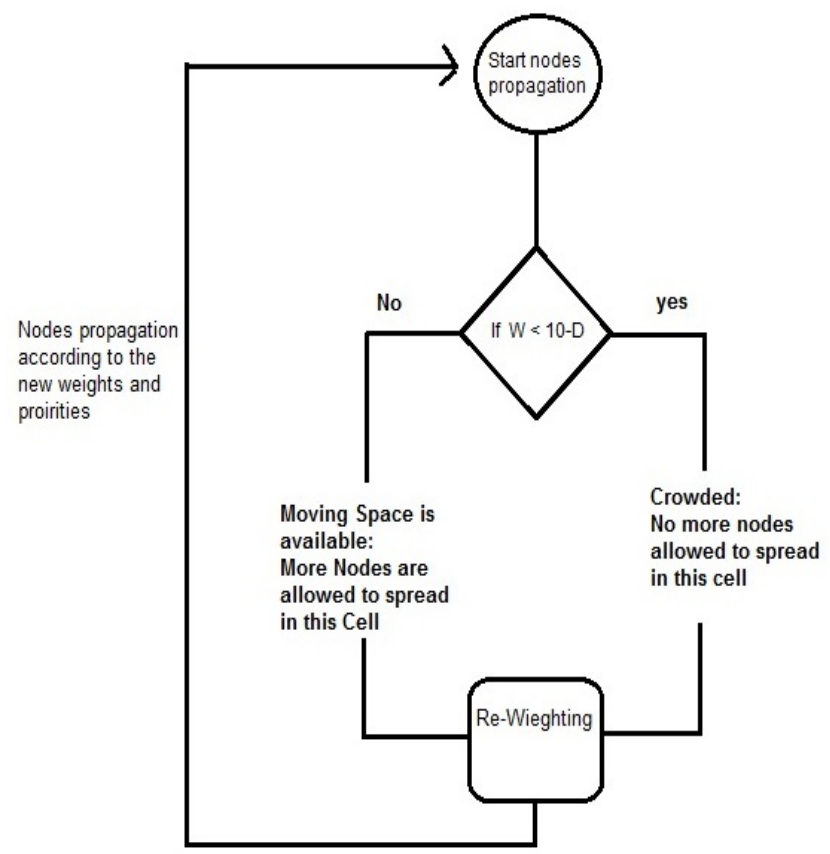

Fig. 4. Dividing the disaster are into hexa-cells

\subsection{The proposed model variables:}

The proposed model equation is calculated using the following variables:

Variable "A": Emergency centers, which is valued by:

- Emergency centers capacity.

- Type of the E-center: Health caring (Value=1), Technical support (Value =2).

- (Priority of Health centers is higher)

- Rank of the center: Counter of the same type of the centers.

A number is given for the first center, and decreased by 1 each time another center of the same type is detected. 
Variable "B": Nearby roads segments

- Number of roads segments.

- Capacity of segments (Width of the street)

- Damage percentage.

Variable "C": Border nodes existed in the border cells of the disaster area (Have the Higher weights).

Considering the above calculated according to their algorithms; the algorithm of the cell weight in which the emergency centers shall be propagated is:

Cell weight $=$ number of nodes $+\mathrm{A}+\mathrm{B}+\mathrm{C}$.

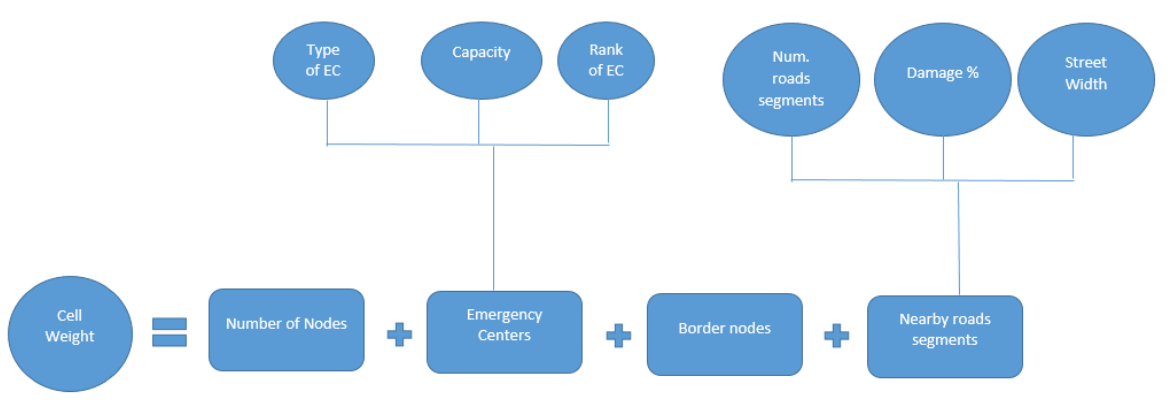

Fig. 5. Proposed model for emergency centers setup

The highest cell weight is considered and emergency center cell in which a health center or a technical support center shall be found.

\section{Conclusion}

The proposed model enables the network to propagate the nodes into the emergency centers in the disaster areas. Those areas are divided into hexa-cells, the centers are categorized as health and technical centers. Then a rank is given for each center. The model takes into account the streets segments, the emergency centers types and ranks, the border cells and nodes, where the priority is given for the health care centers and border cells with their nodes.

For future studies, the mobility model shall be incorporated into used routing mechanisms to evaluate its role in enhancing performance during crises and disasters situations.

\section{$5 \quad$ References}

[1] K. F. Rauscher, (2001). "Wireless Emergency Response Team - Final Report for the Sep. 112001 NYC WTC Terrorist Attack”, Oct. 2001. 
[2] Ray, N., Turuk, A. (2016). A framework for post-disaster communication using wireless ad hoc Networks. Integration the VLSI journal, retrieved on https://doi.org/10.1016/j.vlsi.2016.11.011

[3] Portmann, M., Pirzada, A.A. (2008). Wireless mesh networks for public safety and crisis management applications, IEEE Internet Comput. 12 (1) 18-25. IEEE. https://doi.org/10.1109/MIC.2008.25

[4] Manaseer, S., Alawneh, A. (2017). A New Mobility Model for Ad Hoc Networks in Disaster Recovery Areas. International Association of Online Engineering. V. 13, No.3. https://doi.org/10.3991/ijoe.v13i06.7106

[5] Lee, H., Jeon, D. (2015). A Mobile Ad-Hoc network multipath routing protocol based on biological attractor selection for disaster recovery communication, The Korean Institute of Communications Information Sciences. ICT Express 1 (2015) 86-89. https://doi.org/10.1016/j.icte.2015.10.001

[6] Dressler, F., and Akan, O.B., (2010). A survey on bio-inspired networking, Comput. Netw.54 (6). https://doi.org/10.1016/j.comnet.2009.10.024

[7] Briante, O., Loscri, V., Pace, P., Ruggeri, G., Zema, N. (2015). COMVIVOR: an evolutionary communication framework based on survivors' devices reuse, Wirel. Pers. Commun. 85 (4) 2021-2040. https://doi.org/10.1007/s11277-015-2888-y

[8] Schroder, S., Wagstaff, K., Rogers, S., Langley, P., Wilson, C., (2004). Mining GPS traces for map refinement, Data Min. Knowl. Discov. 9 (1) 59-87. https://doi.org/10.1023/ B:DAMI.0000026904.74892.89.

[9] T. C. Gunaratna, G \& V. N. M. Jayarathna, P \& S. P. Sandamini, S \& S. De Silva, D. (2015). Implementing wireless Adhoc networks for disaster relief communication. https://doi.org/10.1109/UMEDIA.2015.7297430

[10] Ramon, M\& Crowcroft, A \& Yoneki, J \& Martí, E. (2012). Evaluating Opportunistic Networks in Disaster Scenarios. Journal of Network and Computer Applications. https://doi.org/10.1016/i.jnca.2012.11.001

[11] Kikuchi, Y., Shibata, Y. (2015). Mobile cloud computing for distributed disaster information system in challenged communication environment, in: Advanced In- formation Networking and Applications Workshops (WAINA), 2015 IEEE 29th International Conference on, IEEE, pp. 512-517. https://doi.org/10.1109/WAINA.2015.82

[12] Chenji, H., Zhang, W., Stoleru, R., Arnett, C. (2013). Distressnet: a disaster response system providing constant availability cloud-like services, Ad Hoc Netw. 11 (8) 24402460. https://doi.org/10.1016/j.adhoc.2013.06.008

[13] Channa, M. \& Ahmed, K. (2010). Emergency Response Communications and Associated Security Challenges. International Journal of Network Security \& Its Applications. https://doi.org/10.5121/ijnsa.2010.2414

[14] Srivastava, A., Kumar, D. \& S.C. Gupta. (2014). Mobile Ad-Hoc Network Performance in a Disaster Management Scenario. African Journal of Computing \& ICT. Vol.7, No 1.

[15] Nguyen, H., Gyoda, K., Okada, K., Takizawa, O. (2011). 2-2 Ad hoc based emergency communications models for wide-scale disaster management. Journal of the National Institute of Information and Communications Technology. Vol. 58, No.1-2, Pp 95-105.

[16] Manaseer, S., Alsoudi, D., Aljawawdeh, A. (2017). Border node detection: a new experimental approach. Journal of Computer Modelling \& New Technologies, Vol. 21, No. 2, Pp: $37-40$. 


\section{Authors}

Saher Manaseer has a $\mathrm{PhD}$ in Computer Science from the Department of Computing Science at the University of Glasgow. His main area of research is Computer Networks and Embedded Systems. Currently, Dr. Manaseer is an active researcher in the field of Mobile Ad Hoc Networks. More specifically, his research on MANETs is focused on developing MAC layer protocols. Before obtaining his $\mathrm{PhD}$, He got his Masters in Computing Science from the University of Jordan where his research in that era was focused on Software Engineering. Since he earned his undergraduate degree in Computer science, Dr. Manaseer has worked in two parallel directions, being Training and software development.

Afnan Alawneh is a member of Jordan University staff, works in the University of

Jordan library, and as a researcher, quality assurance representative, got her B.Sc. in Information technology in 2005, and completed an MBA in 2014, both form the University of Jordan.

Article submitted 2018-11-13. Resubmitted 2019-01-08. Final acceptance 2019-02-22. Final version published as submitted by the authors. 